\title{
2D FLUID STRUCTURE INTERACTION ANALYSIS OF A VERTICAL AXIS TIDAL TURBINE BLADE USING PERIODIC INFLOW EQUIVALENCE MODEL
}

\author{
Nu Rhahida Arini ${ }^{(*)(* *)}$, Stephen R. Turnock ${ }^{(*)}$, Mingyi Tan ${ }^{(*)}$ \\ (*) University of Southampton, United Kingdom \\ ${ }^{(* *)}$ Electronic Engineering Polytechnic Institute of Surabaya, Indonesia
}

\begin{abstract}
Fluid structure response of vertical axis tidal turbine blades using NACA 0012 and periodic inflow equivalence model is predicted in this work. The response is investigated numerically by developing a 2D CFD model at high Reynolds number $\left(3.07 \times 10^{6}\right)$. The periodic inflow equivalence model is conducted by modelling the rotation of the turbine as time dependent unsteady incoming fluid velocity and angle of attack variation entering the 2D CFD domain. The blade response is modeled by a vibrational system with spring damper components which are attached at the blade fluid dynamic center point. The aim of this study is to predict a resonant condition or a lock-in frequency induced by wake generation at a vertical axis turbine blade during the turbine operation. The model is generated using a dynamic mesh construction in OpenFOAM 2.2 and the mesh is refined using snappyHexMesh utility. The mesh has seven added boundary layers around the blade surface and simulated using k- $\omega$ SST turbulence model. Drag, lift and moment force coefficient are observed during 12 seconds which is equal to 3.3 revolutions and extracted using Fast Fourier Transform method to obtain its predominant frequency. The predominant frequency determines the dynamic condition of the blade and is used for predicting a resonance based on the turbine's natural frequency. The result shows that the vertical axis tidal turbine which is manufactured from a composite with material pitch stiffness of $200 \mathrm{Nm} /$ radians, heave stiffness of $1000 \mathrm{~N} / \mathrm{s}$, and operates at tidal velocity of $0.656 \mathrm{~m} / \mathrm{s}$ is found to experience a resonance or lock-in phenomena induced by wake generation in the pitch mode response.
\end{abstract}

\section{Introduction}

Renewable energy has been widely used as an energy alternative not only because of the depletion of fossil fuel but also because it is environmental friendly. However some devices still needs to be developed including vertical axis tidal turbines. Although a vertical axis tidal turbine has been examined for decades but its interaction with the fluid passing through the turbine has not been explored and understood. Therefore the aim of this work is to investigate in 2D the fluid structure response (FSI) of a vertical axis tidal turbine blade using a time varying incoming velocity and angle of attack model which is equivalent to the vertical axis tidal turbine periodic revolution. This allows the development of a strip theory based approach for estimating the vertical axis tidal turbine blade fatigue and the influence of structure design solutions. The aim is accomplished by developing a suitable CFD model for the time varying angle of attack and incoming velocity for a typical vertical axis tidal turbine and hence to evaluate the FSI response for a representative set of damping constant for a typical composite material.

A vertical axis tidal turbine extracts energy from the kinetic energy of tides and converts it to mechanical energy form by turbine rotations. Unlike wind turbines which have been developed thoroughly, vertical axis tidal turbines still need to be explored and improved to gain better performance. Lundin (2010) mentions several designs which are commonly used for capturing marine and tidal current energy including barrages and turbines. Rouke (2009) reviewed tidal turbine technologies and identified the challenging issues and future development of tidal turbines which were more advantageous than barrage type and produce less pollutants. A vertical axis tidal turbine was also investigated by Almohammadi et al. (2015), however their result only showed the fluid regime in three azimuth angles and torque performances. The blade interaction and the vibration are not yet observed.

In many designs, a vertical axis tidal turbine is arranged symmetrically with two, three or four blades and it is assumed that all blades experience same forces and generated same responses. The forces work on a turbine blade introduced by the tide flows, induce lift, drag, and moment forces. In a symmetric vertical axis turbine, blades are assumed to experience lift, drag and moment forces similarly thus only one blade instead of all blades is modelled and observed in this work. The tide velocity is also assumed constant and fluid load is uniformly distributed along the spanwise length of the blade.

As a blade contacts with the tides, a destructive vibration is excited on a turbine structure and leads to fatigue failure which can reduce turbine life time and performance. The vibration can also generate a singing phenomenon which may potentially harm the environment. Controlling vibration is of importance to minimize the risks and one way to do so by predicting vibration frequency lock-in on the turbine blade which is reliably obtained from a blade response analysis. The analysis is conducted by modeling the turbine blade response as a first mode 
vibrational system. The model is constructed as a 2D CFD vibrational spring damper system which are attached at fluid dynamic center point on blade chord line. Earlier works for analyzing the loading in a vertical axis tidal turbine has been done such as works by Young (2010), Banks (2013), Lanzafame (2014), Brusca (2014) and Gretton (2009). However the response of the turbine blades in terms of blade vibration has not well understood and observed. Predicting the turbine response is significant to avoid destruction from a resonance during turbine operation and enhance turbine life time and performance.

The progressive development of computer power helps engineering problems to be solved faster and give more precise result. Since the last decade many fluid dynamic problems have been investigated by the implementation of CFD including in vertical axis turbine area. Gretton (2009) developed a model of vertical axis tidal turbine and started his studied by modeling a single fixed angle of attack blade. He continued with an oscillating blade and finally built a model for three bladed NACA 0012 vertical axis tidal turbine. He run his model in k- $\omega$ SST turbulence model. He found the tidal turbine performance as a function of tip speed ratio and azimuth angle. Lanzafame (2014) developed a 2D CFD model for vertical axis tidal turbine using transition turbulence model. The model used a hybrid mesh which comprised of a structure grid in the blade vicinity and unstructured grid in the far field region. The far field region was a rectangular fixed domain in which the rotating turbine domain was placed. Some boundary conditions (BC) were defined including inlet, outlet, and symmetry for top and bottom part, wall and interface BC. The interface was the contact surface between the fixed domain and rotating turbine domain. Brusca (2014) and Gosselin (2013) studied relations of some parameters for designing a vertical axis turbine. Brusca (2014) studied the influence of turbine aspect ratio to power coefficient using Multiple Stream Tube Model. Gosselin (2013) conducted a parametric study for some vertical axis tidal turbine parameters to design a turbine numerically and simulated using k- $\omega$ SST turbulence model. The parameters which are solidity, number of blades, Reynolds number , pitch angle (fixed and variable), and blade thickness are varied to obtain the best hydrodynamic configuration. He also simulated and compared three different turbulence models which were spalart almaras, $\mathrm{k}-\omega \mathrm{SST}$, and transition SST. His result showed that $k-\omega$ SST was the most robust and gave more consistent result amongst those three.

The use of computers in CFD has been proven to give an accurate and quick prediction to solve fluid dynamic problems. In the area of fluid structure interaction, CFD has also been a promising choice for solving many problems. Khalid (2013) studied a numerically coupled method for a four bladed vertical axis tidal turbine dynamic response with unsteady incoming flow and validated with his own experiment. He used two ways FSI methods which solve hydrodynamic and structural problem iteratively and later he found that his simulation gave a precise and accurate result with the experiment. His simulation result showed the velocity distribution profile, the prominent stress and deformation on the each blade spanwise for various turbine angular velocities. The importance of blade deformation on turbine operation initiated dynamic response in a turbine operation was studied by Banks (2013). Banks numerically investigated a better method to calculate turbine blade deformation by comparing BEM and LES methods. He applied fluid converged solutions to a finite volume approach to find the thrust coefficient and deformation on the blade span. His simulation revealed that a flapwise deformation which was essentially influenced by turbine operations, could not be neglected in BEM method. Li (2010) suggested four characteristics for a turbine including power output, torque, induced velocity, and emission to facilitate the development of standards for tidal current turbine. Bessem (2015) proposed a model for free vibrating airfoil with two springs for an increasing and decreasing frequency. He found that the amplitude at lock-in frequency reached four times than unlock frequency. The income fluid flow was varied untill $50^{\circ}$ and a support was attached which allowed the airfoil to rotate with maximum angle of attack of $5^{\circ}$. Young (2010) promoted a numerical method combination of boundary element and finite method and validated it with published experiments. The method investigated thrust coefficient, power coefficient and stress and deflection distribution as a response of fluid loading in tidal turbine.

\section{Response Model}

\subsection{Vibration System for Response Model}

A vertical axis tidal turbine blade response is simulated using a vibration model in this paper. A vibration system with spring and damping components has been used to model a foil response as proposed by Akcabay et.al (2015a, 2015b), Ducoin $(2009,2013)$ and Rana (2011). Akcabay et.al (2015a, 2015b) suggested a model for representing a flexible hydrofoil response using a spring damper system. Their blade was allowed to move in pitch and heave direction in respect to incoming inlet fluid. His result came with a good agreement with Ducoin's result (2009). Ducoin (2013) also applied a vibrational method with a spring damper system to model a hydrofoil response generated by fluid cavity.

The vertical axis tidal turbine blade is allowed to move in heave and pitch mode in respect to incoming tidal velocity which induce transversal and torsional vibration. The vibrational system is modelled with two spring damper systems attached on the turbine blade to assist transversal and rotational vibration as shown in Figure 1 . The model is simulated in OpenFOAM 2.2 using sixDoFRigidBody pointDisplacement to handle the blade motion and run using 
pimpleDyMFoam solver [openFoam, 2016]. The spring and damper are attached the fluid dynamic centre point which is located at a quarter length of chord line from leading edge.

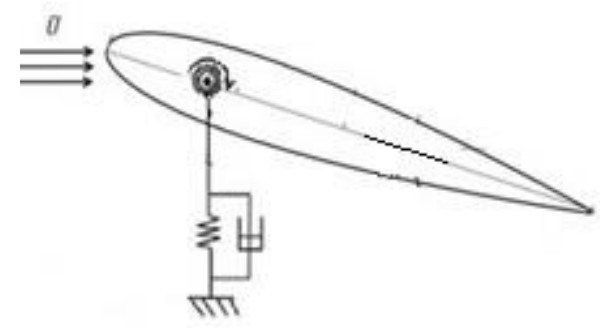

Figure 1. Illustration of a response model as a vibrational system with $U$ is incoming fluid velocity

The torsional and transversal vibration response is assumed first mode of force vibration and performed at quasi steady stage. The simulation is solved using fully coupled FSI method which solve the fluid field and structure equations simultaneously as developed by Chen at.al (2004). The governing equation for the transversal and torsional response are written in Equation 1 and 2

$m \ddot{y}+C_{t} \dot{y}+K_{t} y=L$

$I \ddot{\theta}+C_{r} \dot{\theta}+K_{r} \theta=M$

$\mathrm{m}$ and I denotes blade mass and blade moment of inertia respectively. $\boldsymbol{y}, \dot{\boldsymbol{y}} \ddot{\boldsymbol{y}}$ are displacement, velocity and acceleration in vertical direction performing transversal vibration characteristic. Same superscript symbols apply to $\theta$ representing displacement, velocity, and acceleration for torsional vibration. $\mathrm{C}$ and $\mathrm{K}$ are damping and stiffness coefficient respectively with $t$ and $r$ subscript denoting for transversal and torsional vibration. $L$ is the lift force, works on the blade whereas $\mathrm{M}$ is the moment force.

\subsection{Stiffness and Damping Constant}

The stiffness and damping characteristic of the blade material are taken for transversal (heave) and torsional (pitch) vibration. The stiffness coefficient is calculated using Equation 3 and 4 to find the structure's natural frequency (Equation 5 and 6). The damping and stiffness coefficient is determined based on the blade material which is manufactured from sixty percent of eglass and forty percent epoxy. The typical material composition is proposed to strengthen turbine blade which come from the properties of eglass.

The transversal stiffness constant is adopted from Davies et.al (2013) result which was obtained from an experiment for tidal turbine blades and found that the static flexural stiffness for transversal vibration of blades using polymer was $1000 \mathrm{~N} / \mathrm{m}$. For the torsional vibration, the blade material is assumed to be isotropic which is independent of the blade's orientation thus the stiffness are identical in all directions. However the stiffness constant depends on the mass and moment inertia of the foil. The torsional stiffness coefficient is approximated from the transversal stiffness constant using the ratio of the mass and the moment inertia. The foil mass is calculated using the material density and the foil volume. The model mass which has $0.12 \mathrm{~m}$ spanwise length with the foil shape cross section is found to be $16.73 \mathrm{~kg}$ and the moment inertia in respect with pitch motion is $3.75 \mathrm{kgm}^{2}$. From the isotropic approach using the mass and moment inertia ratio, the torsional stiffness is found to be 224.15 $\mathrm{Nm} / \mathrm{rad}$ and rounded to be $200 \mathrm{Nm} / \mathrm{rad}$. The torsional stiffness constant calculated from the isotropic material approach is verified with torsional stiffness constant calculated using ADM method investigated by Tabassian (2013).

The torsional vibration stiffness coefficient obtained from Tabassian's model (2013) approach is approximated from the natural frequency of a shaft having a circle cross section area and shear modulus of the turbine blade material. He also proposed a torsional analysis to determine a natural frequency for shafts with different supports. One of Tabbassian's shaft models is concentrated mass shaft supported with springs at both ends. The non-dimensional parameters of frequency from Tabbassian's model for a constrained shaft with both ends supported with a spring is 0.808675703 . The length of turbine $(\mathrm{I})$ which is calculated from the blade aspect ratio of 25 (considered from Gosselin 2013 and Brusca 2014) and the blade chord length of $0.75 \mathrm{~m}$ is found to be $18.75 \mathrm{~m}$. The shear modulus which is taken from the loss modulus of epoxy from Chung (2001) is found to be $0.11 \mathrm{GPa}$. The torsional stiffness constant obtained using this method is found to be $278.42 \mathrm{Nm} / \mathrm{rad}$ which is close to the torsional stiffness constant obtained from the isotropic approach. The discrepancy of torsional stiffness coefficient between the ADM method and isotropic approach is likely because in the ADM method the observed shaft had a circular cross section area. 
The importance of damping for response model was studied by Villeaux and Dumas (2013). They applied a damping factor to combined pitch and heave motion on a NACA 0012 model. They developed the model and validated it with their own experiments. Within a range of heave stiffness their model with damping effect applied was found to give closer result to the experiment than a case without damping. In this work, a damping factor is also implemented based in Villeaux and Dumas (2013) result and applied on the response model using spring and damper system.

Another advantage in the use of composite materials is the ability to absorb vibrational energy. Kumar (2015) conducted an experiment to determine the damping characteristics of hybrid polymer matrix composites. He found that the damping ratio directly proportional to natural frequency (Table III of his paper). The damping ratio of the model in this work is found to be 0.0074 with a critical damping value in heave direction is $258.69 \mathrm{Ns} / \mathrm{m}$. The critical damping is calculated using Equation 3.

$$
\mathrm{C}_{\mathrm{c}}=2 \sqrt{\mathrm{mK}}
$$

Where $C_{c}$ is critical damping, $m$ is the foil mass, and $K_{t}$ is transversal stiffness

The damping coefficient is calculated using Equation 4:

$$
\zeta=\frac{\mathrm{C}}{\mathbf{C}_{\mathbf{c}}}
$$

Where $\zeta$ is damping ratio and $\mathrm{c}$ is damping coefficient.

The damping coefficient in heave direction calculated using Equation 4 is found to be $2 \mathrm{Ns} / \mathrm{m}$. The method for determining heave damping coefficient is also applied to the torsional damping coefficient calculation and the damping ratio from Kumar (2015) is employed. The torsional critical damping calculated using equation 3 with an adjustment for the foil mass and transversal stiffness coefficient to be substituted by foil moment inertia and torsional stiffness coefficient respectively. The rotational critical damping is found to be $50 \mathrm{Ns} / \mathrm{m}$ and inserted into equation 4 to determine the torsional damping coefficient. The torsional damping coefficient is found to be 0.2 $\mathrm{Ns} / \mathrm{m}$.

\subsection{Natural Frequency}

Natural frequency $(\omega)$ is a mechanical property of an undamped material associated with its fee vibration frequency when the material is initally displaced. From equation 1 and 2 when the blade model is undamped, only mass and stiffness component are remained and all right hand side are zero. The equations are second order harmonic differential equation and the solution is satisfied by finding a matrix for $\omega$ as an eigenvalues. Thus in simple manner natural frequency can be calculated for transversal and torsional vibration using Equation 5 and 6 .

$\omega_{t}=\sqrt{\frac{K_{t}}{m}}$
$\omega_{r}=\sqrt{\frac{K_{r}}{I}}$

$\omega$ denotes natural frequency with $t$ performing transversal vibration and $r$ for torsional vobration. $\mathrm{K}_{\mathrm{t}}$ and $\mathrm{K}_{\mathrm{r}}$ value are taken from the transversal and torsional material stiffness which has been explained in the previous section. Both values are applied to Equation 5 and 6 to determine the transversal and torsional natural frequencies. The transversal and torsional natural frequency are found to be $0.9425 \mathrm{~Hz}$ and is $1.4758 \mathrm{~Hz}$.

\section{Numerical Model}

A 2D CFD model of vertical axis tidal turbine NACA 0012 blade is developed with a structured grid using snappyHexMesh utility to refine the mesh around the blade surface as shown in Figure 2. Seven boundary layers are built around the blade and three refinement regions are added with two surface adjustment between layers. The boundary layer is a quadrilateral structured layer generated over the foil surface. It is extruded from the foil surface in the normal direction of the foil. Number of cells are same for each layer in structured grid. This gradually increases cell shapes from the first to the uppermost layer. The domain is a rectangular mesh which has 12402 cells in total as listed in table 1. 
Table 1. Mesh statistic of the model

\begin{tabular}{|c|c|}
\hline parameter & Number \\
\hline \hline points & 25822 \\
\hline faces & 24860 \\
\hline cells & 12402 \\
\hline
\end{tabular}

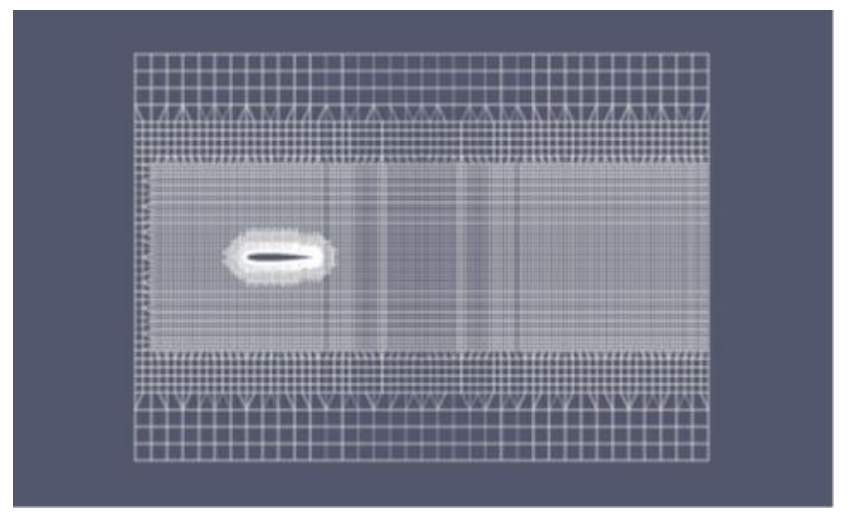

a.

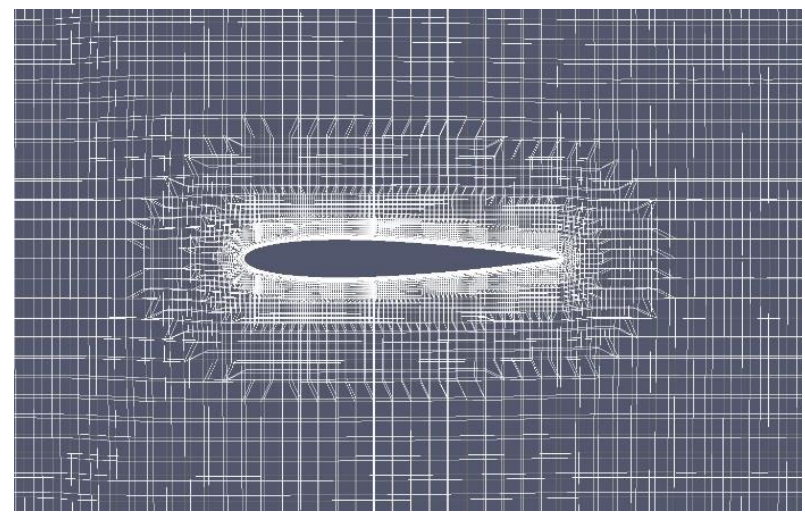

b.

Figure 2. a. A blade model domain, b. The blade refinement mesh

The CFD model employs k- $\omega$ SST turbulence model and pimple algorithm to be applied in the blade dynamic mesh. The blade uses NACA 0012 with $0.75 \mathrm{~m}$ chord length and located at $2 \mathrm{c}$ from domain inlet which has total length of 7c. Courant number is 0.9 and residual coefficient for all properties are $10^{-5}$. The basic simulation set up is using zero degree for initial angle of attack and is allowed to move in two degree of responses in pitch and heave. The blade stiffness coefficient of $1000 \mathrm{~N} / \mathrm{m}$ and $200 \mathrm{Nm} / \mathrm{radian}$, and blade damping coefficient of $2 \mathrm{Ns} / \mathrm{m}$. Tidal velocity is assumed constant and taken from the maximum velocity which has been recorded at East Indonesian sea as the case study in this work (Tillinger, 2011). The model takes the maximum tidal velocity to investigate the extreme condition experienced by the turbine which is likely to generate the stronger vibration than the lower tidal velocity magnitude.

\section{Periodic Inflow Equivalence Model}

Under operation, vertical axis tidal turbine blades experience two modes of responses which are turbine rotation and structural vibration. The turbine rotation is a means to capture tidal energy and converts it into a mechanical energy to rotate a generator. Meanwhile the vibration is caused by interaction of blade with unsteadiness of fluid flow. In a dynamic motion point of view, an axis of motion determine the motion orientation and thus the equation of motion which should be applied. Two different modes with different axis of motion make a response blade model complicated to generate and also make the model time consuming to run. To overcome this situation, a method is proposed, named Periodic Inflow Equivalence, to model the rotational response of vertical axis turbine by employing time varying rotational conditions to the time varying incoming fluid velocity entering the domain in the 2D CFD model.

The periodic inflow equivalence model develops a time varying angle of attack and fluid resultant velocity into an equivalent periodic changing inflow passing through a static initial angle attack blade. This method allows only one blade to model the response motion. The periodic inflow equivalence model develops a time dependent mathematical model for a turbine rotational velocity and applies it to incoming velocity to express the turbine rotation. In this case the turbine vibration visualizes structural vibration only without a rotational motion. In this method, the earth reference coordinate is located on the blade and imaginary moves together with the blade and follows the blade rotation.

The concept of the periodic inflow equivalence model can be explained using Figure 3. A turbine rotation influences the magnitude and direction of incoming fluid velocity as illustrated in Figure 3a. The incoming velocity which works on a turbine blade is a resultant velocity between tidal velocity and angular velocity of a turbine. The incoming fluid has time dependent velocity variation at all points of a turbine revolution and locates between certain ranges of angle attack as illustrated in Figure 3b. Gosselin (2013) proposed an equation to define time varying angle of attack for a rotating blade of vertical axis tidal turbine as written in equation 7

$\alpha=\arctan \left(\frac{1}{\lambda \cdot \sin (\theta)}+\frac{1}{\tan (\theta)}\right)+\theta-\frac{\pi}{2}$ 
Where $\alpha$ is the angle of attack, $\lambda$ is turbine tip speed ratio (TSR), and $\theta$ is the azimuth angle. Tip speed ratio is defined as the ratio between tangential velocity working on a turbine blade and free stream velocity. Equation 7 is used to determine the time dependent velocity variation of vertical axis tidal turbine using the typical turbine and fluid characteristic as listed in Table 2. Another turbine parameter which is of importance is solidity as also shown in Table 2. Solidity is defined as the ratio of the area swept by turbine blade to the total area through which incoming fluid pass. Details for determining the turbine characteristics as listed in Table 2 can be found in Gosselin (2013).

Table 2. Turbine and fluid characteristic

\begin{tabular}{|l|r|}
\hline Tip Speed Ratio & 5.1 \\
\hline Solidity & 0.1829 \\
\hline Radius $(\mathrm{m})$ & 1.957893 \\
\hline kinematic viscosity (m2/s) & $9.78 \mathrm{E}-07$ \\
\hline density $(\mathrm{kg} / \mathrm{m} 3)$ & 1025 \\
\hline dynamic viscosity $(\mathrm{kg} / \mathrm{m} . \mathrm{s})$ & $1.00 \mathrm{E}-03$ \\
\hline Turbine angular velocity (rad/s) & 1.71 \\
\hline
\end{tabular}

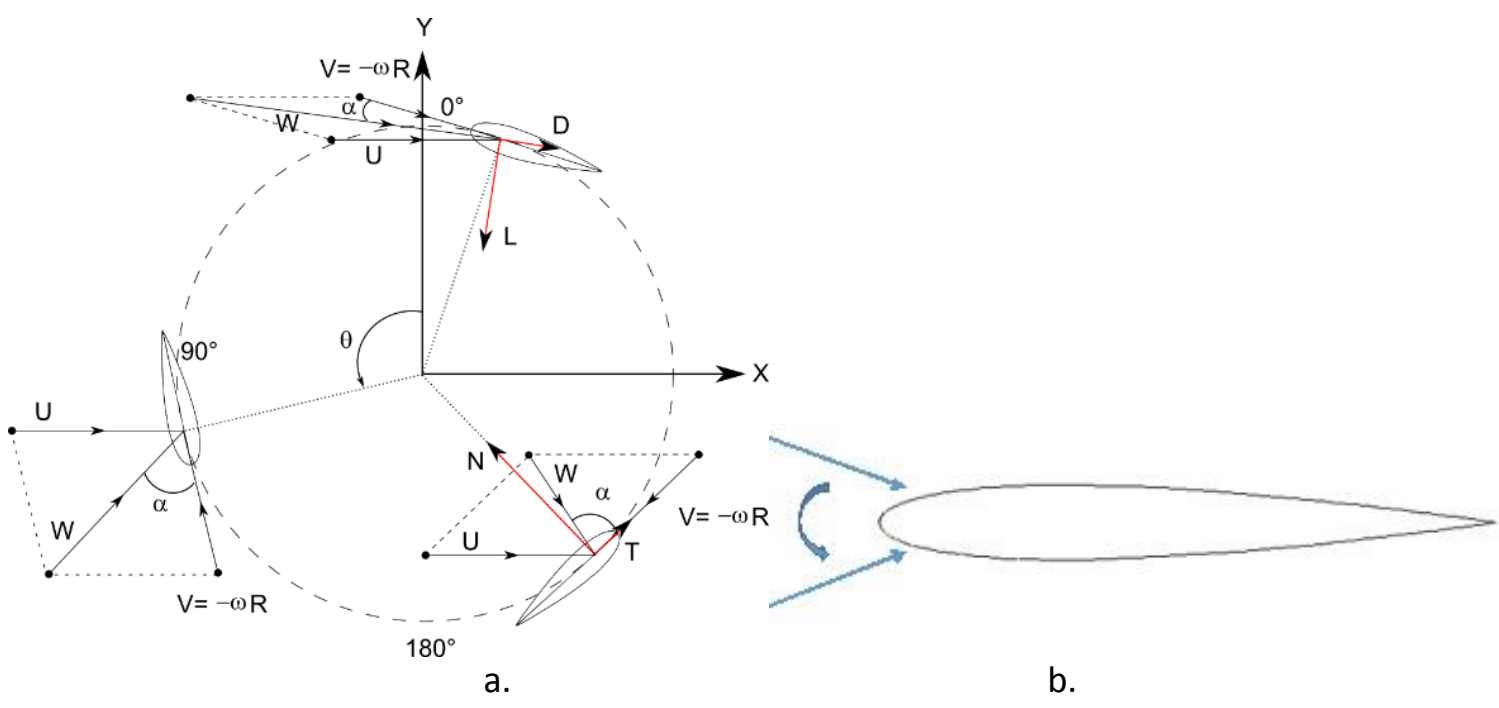

Figure 3. a. illustration of time varying incoming fluid velocity and angle of attack over one revolution of a vertical axis turbine $(\omega=$ turbine angular velocity, $\theta=$ azimuth angle, $\alpha=$ angle of attack, $D=$ drag force, $L=$ lift force, $\mathrm{U}=$ tidal velocity, $\mathrm{W}=$ resultant velocity, $\mathrm{V}=$ turbine tangential velocity, $\mathrm{T}=$ thrust force, $\mathrm{N}=$ normal force), b. schematic of pitch and angle of attack equivalence model of a vertical axis tidal turbine blade

\section{Mesh Independence Study and Validation}

Mesh independence study is applied to four different refinement grids. The four different grids have 11565 cells, 12402 cells, 18042 cells and 29120 cells. The study compares the drag force at $8^{\circ}$ static angle of attack as shown in Figure 4.

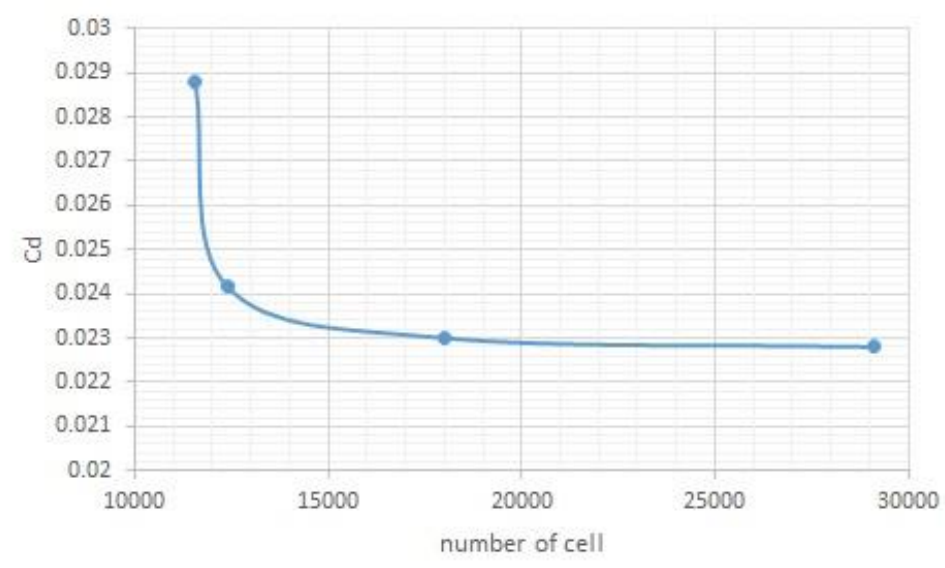

Figure 4. Drag coefficient at four different refinement grids 
From figure 4, it can be seen that Cd value is drastically reduced at the second refinement grid with 12402 cells and closer to Abbott's experimental data. From the second refinement, the more refined grid does not influence the $\mathrm{Cd}$ value. The execution time of higher refinement grid is also affected. The grid with 29120 cells is simulated nine times longer than the first refinement. Based on the result of the mesh independence study, the second refinement with 12402 cells is utilized in this work. The force coefficients of the model with chosen grid refinement is further validated using the reference data. The validation of lift and drag coefficient to the reference data are shown in Figure $5 a$ and $5 b$ respectively.

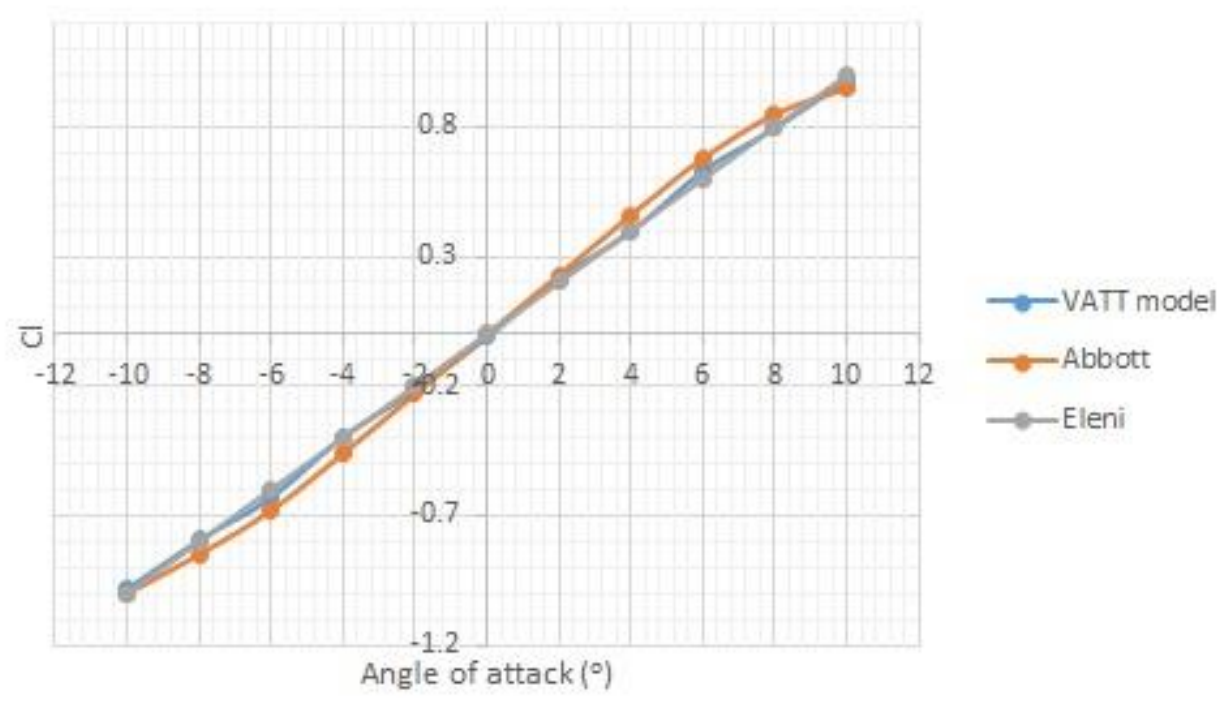

a.

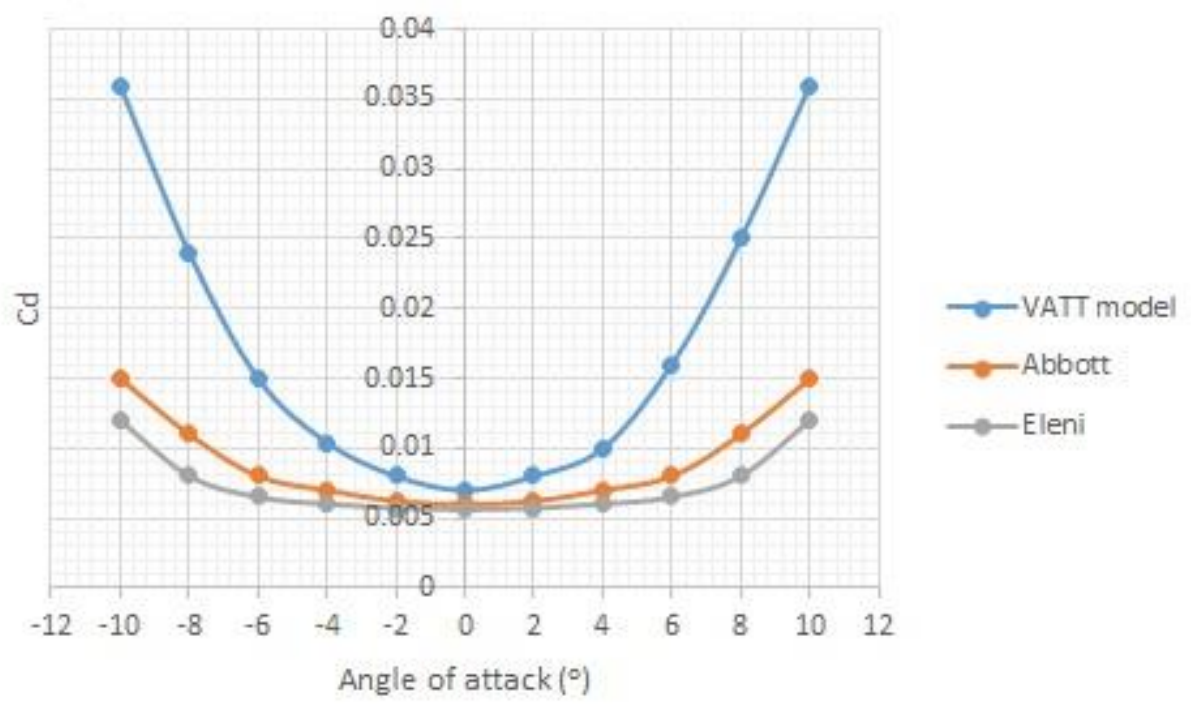

b.

Figure 5. Forces coefficient of the model and reference data from Abbott (1955) and Eleni (2012): a. Lift coefficient, b. Drag coefficient

The $\mathrm{Cl}$ result shows agreement to Abbott and Eleni's data with the highest error of $8 \%$ occuring at $6^{\circ}$ angle of attack. For drag coefficient in Figure $5 b$, the error is fairly high due to the lack capability of the mesh solving the viscous effect of the model. The viscous effect dominates the drag force in the boundary layer. The requirement of a refined mesh to attain accurate drag force may not be reliable for the dynamic mesh solution. A dynamic mesh requires a robust mesh boundary layer with adequate number of cells generation. The number of cells is a critical aspect in the dynamic mesh modelling as to affect the computer simulation time for solving the changing mesh configuration in each time step. Thus a compromise should be taken into account in dynamic mesh modelling including this vertical axis tidal turbine response model.

This mesh topology is further generated for the vertical axis tidal turbine blade model and simulated using pimpleDyMFoam solver of OpenFOAM 2.2. The mesh is run using PIMPLE algorithm and dynamic mesh configuration which manage mesh motion and grid topology changes due to FSI turbine response. The strip theory is applied on the spanwise length of a turbine with the FSI analysis is being introduced on the middle of the spanwise length. The 
mathematical model of fluid continuity and momentum equation coupled with kinematic motion equation of spring damping system are assigned and executed in the cells within the dynamic mesh.

Validation is also conducted on the dynamic mesh configuration. The dynamic mesh validation is applied to the vertical axis tidal turbine response model at three selected azimuth angles. These are $0^{\circ}, 120^{\circ}$ and $240^{\circ}$. The pressure contour plot at the selected locations are shown in Figure 6 . The fluid regime and the pressure contour in those locations are verified with the result from Almohammadi et al. (2015).

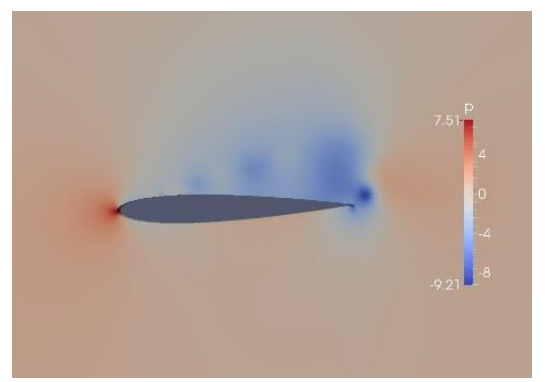

a.

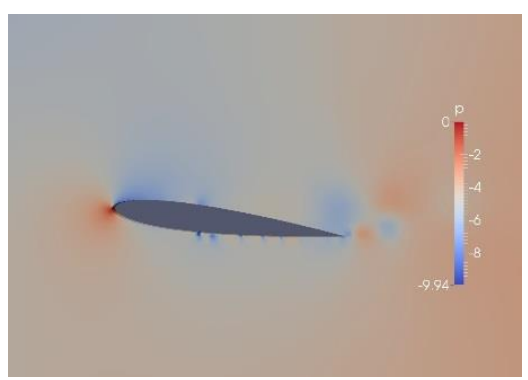

b.

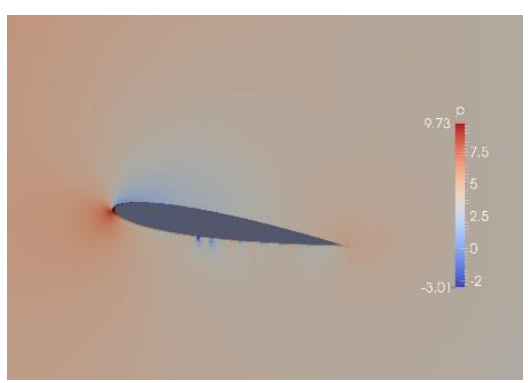

c.

Figure 6. Pressure contour at three selected turbine blade positions of azimuth angle: a. $0^{\circ}$, b. $120^{\circ}$, c. $240^{\circ}$.

The fluid regime at three locations are satisfied with Almohammadi et.al (2015) results. At $0^{\circ}$ azimuth angle, the fluid is attached on both surfaces of the foil. The vortex is shown to be released on the top of the foil and behind the trailing edge. At azimuth angle of $120^{\circ}$, the vortex is shed and can be detected from pressure coefficient distribution as explained by Lee and Su (2015). The vortex generation is indicated by pressure coefficient contour peak in the pressure coefficient plot in Figure 7. At $0^{\circ}$ azimuth angle, the leading edge vortex has flowed to the trailing edge and released to the free stream which is also shown in Figure 6a. At azimuth angle of $120^{\circ}$, Leading Edge Vortex (LEV) is shed as marked by peaks at $x / c=0.39$ in Figure 7. In this state the fluid regime is chaotic as a result of dynamic stall and fluid separation. At $240^{\circ}$ azimuth angle the pressure coefficient plot is flat and no peaks is found in which indicates that no vortex is generated. The fluid regime in other locations will be detailed in Section 6.3

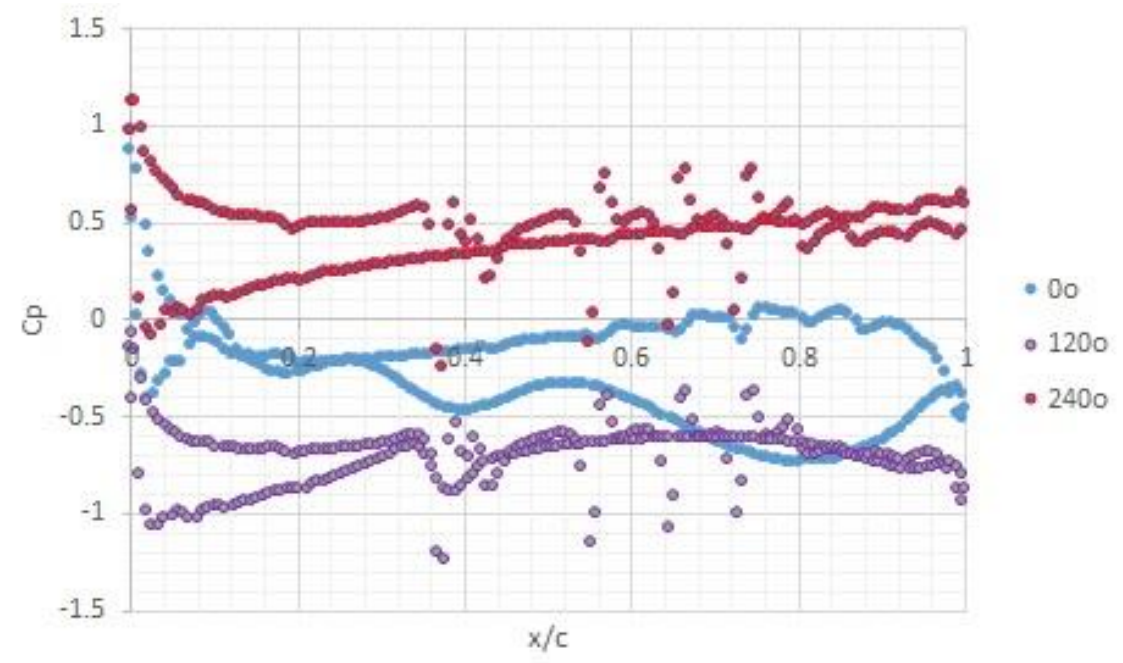

Figure 7. Pressure coefficient distribution at selected azimuth angle $0^{\circ}, 120^{\circ}$, and $240^{\circ}$

\section{Result and Discussion}

The time varying angle of attack over one turbine revolution which is calculated using Equation 7 is depicted in Figure $8 \mathrm{~b}$ and the incoming fluid velocity on one revolution is shown in Figure 8a. This value is employed to incoming velocity in the model using groovyBC utility which is applied in the $U$ Dictionary under the 0 folder in OpenFOAM for velocity description. To model the vibration response, sixDoFRigidBodyDisplacement is assigned in the blade condition in the pointDisplacement Dictionary. 


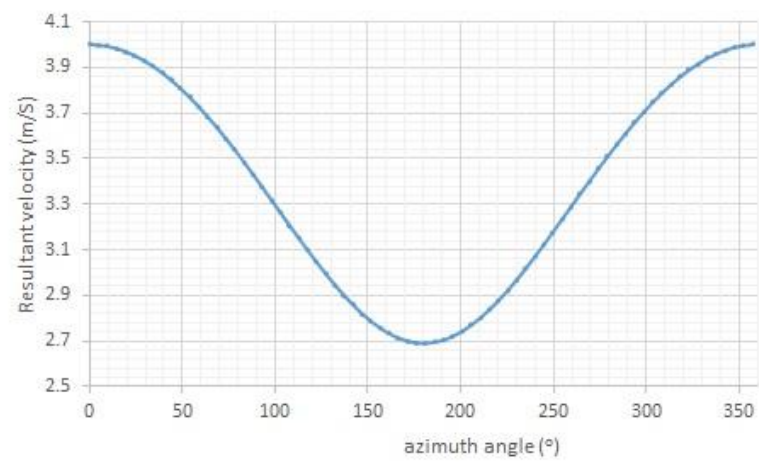

a.

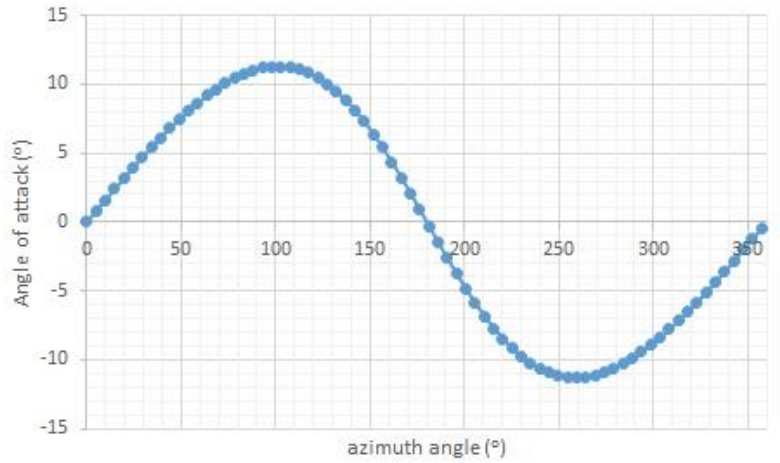

b.

Figure 8. a. Resultant velocity over one revolution of a turbine, b. angle of attack over one revolution of a turbine

From Figure $8 \mathrm{a}$ and $8 \mathrm{~b}$, it can be seen that the changing angle of attack and velocity are periodic. This periodicity is then used to define the flow passing through the blade. It is also noticed that the incoming velocity reaches maximum at $0^{\circ}$ or $360^{\circ}$ azimuth angle, in contrast with the angle of attack which is reaches minimum in this location. The combination of the time varying angle of attack and velocity effects to fluid behavior as indicates in the periodic forces coefficient and near wake profile.

\subsection{Forces Coefficient.}

The result of time dependent drag, lift, and moment force coefficient are illustrated in Figure 9a, 10a, and 11a for fifteen seconds simulation time or equals to 4.1 turbine revolutions. The frequency domain plot with respect to the time domain plot of the three forces which are extracted using Fast Fourier Transform method are given in Figure $9 b, 10 b$, and $11 b$.

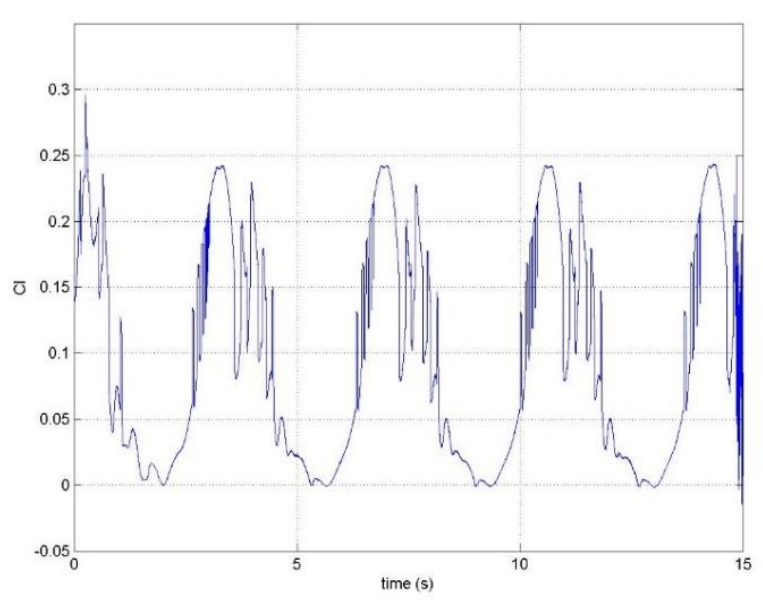

a.

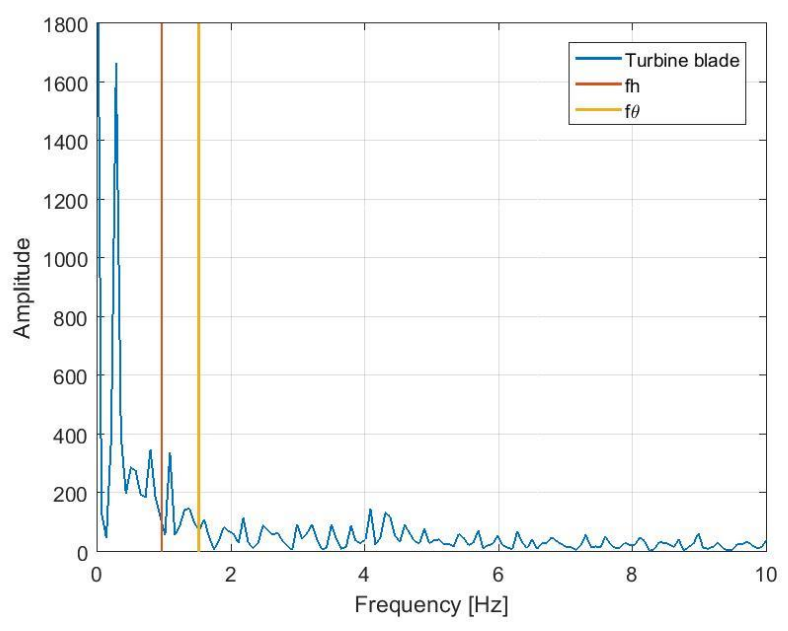

b.

Figure 9. Lift force: a. time dependent, $b$. frequency domain. $f_{h}=$ blade's natural frequency in heave direction, $f_{\theta}=$ blade's natural frequency in pitch direction

From figure 9a, 10a, and 11a it can be seen that simulation gives transient results at the first three seconds approximately. As calculated from data in Table 2, angular velocity of the turbine is found to be $1.7 \mathrm{rad} / \mathrm{s}$ and thus for one revolution, the turbine rotates for 3.7 seconds. During twelve seconds steady period, the turbine has 3.3 cycles as can be seen from those three pictures of time dependent force coefficients. The pictures show the complete three repeating peaks during the 12 seconds time recorded. Maximum fluid loading exerted by maximum resultant velocity happens at zero azimuth angle and zero initial angle of attack when turbine tangential velocity is parallel to the tidal velocity. The minimum fluid loading appears when azimuth angle is $180^{\circ}$ and tangential velocity parallel to tidal velocity but flows in the opposite direction. Accordingly for first half of turbine revolution (from zero azimuth angle until $180^{\circ}$ ), the angle of attack is moving half cycle of angle of attack range, as illustrated in Figure $8 \mathrm{~b}$, from zero degree to $12^{\circ}$ and decreasing back to zero initial angle when azimuth angle is $180^{\circ}$. At azimuth angle of $180^{\circ}$ the incoming velocity reaches minimum. At the second half of turbine revolution, initial angle of attack moves from zero degree to -12 degree and after reaches the minimum angle, the angle of attack moves back, and reaches zero degree at $360^{\circ}$ azimuth angle where the resultant velocity is minimum. The point where maximum and 
minimum resultant velocity occur has about $180^{\circ}$ azimuth angle difference as illustrated in Figure $8 \mathrm{a}$. This condition causes the lift coefficient periodicity as shown in Figure 9a. The maximum lift coefficient value is 0.24 and occurs at $325^{\circ}$ azimuth angle. The minimum lift coefficient value is zero at $186^{\circ}$ azimuth angle.

From Figure $9 a$ it can be seen that at the maximum lift coefficient point onward, the lift force decreases as the azimuth angle become higher making higher initial angle of attack, thus produces vortex shedding on the surface of the blade. Some fluctuations appear before the lift force reaches minimum at the minimum point. The fluctuation occurrence is also a result of wake generation on the outer surface of a vertical axis tidal turbine blade as the angle of attack varies during turbine operation. Although the angle of attack is symmetric on the two half of turbine cycles, the lift force appears to be asymmetric due to the effect of wake generation on the surface of the blade. At the first half of blade revolution, the angle of attack is positive which means the tidal velocity is parallel to tangential velocity hence enhance the wake generation and produce unsteadiness in the flow. At the second half of revolution, after the azimuth angle reaches $180^{\circ}$, the angle of attack becomes negative and wake generates on the inner surface of the blade. At this blade section, vorticity is enhanced and the lift force becomes unstable and fluctuating. The history of wake generation during a turbine revolution can be seen in Figure 12. The time domain lift coefficient in Figure 9a is further transformed to the frequency domain using FFT as shown in Figure $9 b$ to identify the predominant frequency of the turbine.

From Figure 9b, it can be seen that predominant frequency of lift coefficient which is $0.3 \mathrm{~Hz}$ does not coincide with the natural torsional and transversal frequency. Although the torsional natural frequency lies between two $\mathrm{Cl}$ frequencies of $0.8 \mathrm{~Hz}$ and $1.2 \mathrm{~Hz}$, but these frequencies' power are small hence apparently resonance vibration response does not occur. For the drag force trend, time domain and the frequency domain of $\mathrm{Cd}$ are depicted in Figure 10a and 10b.

From Figure $9 \mathrm{~b}$, it can be seen that predominant frequency of lift coefficient which is $0.3 \mathrm{~Hz}$ does not coincide with the natural torsional and transversal frequency. Although the torsional natural frequency lies between two $\mathrm{Cl}$ frequencies of $0.8 \mathrm{~Hz}$ and $1.2 \mathrm{~Hz}$, but these frequencies' power are small hence apparently resonance vibration response does not occur. For the drag force trend, time domain and the frequency domain of $\mathrm{Cd}$ are depicted in Figure 10a and 10b.

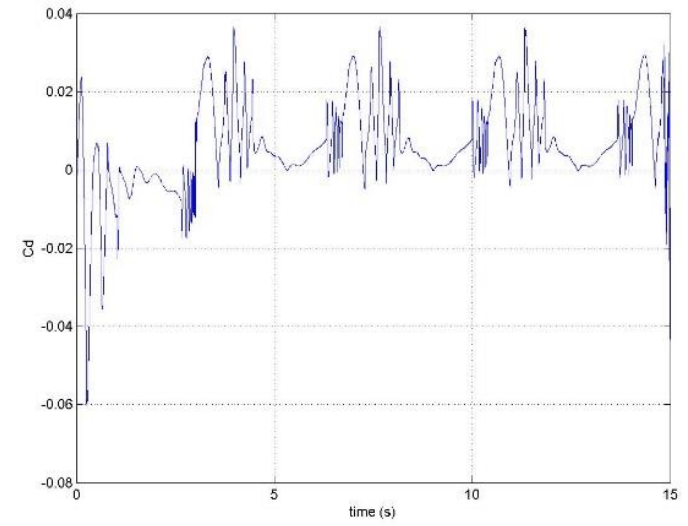

b.

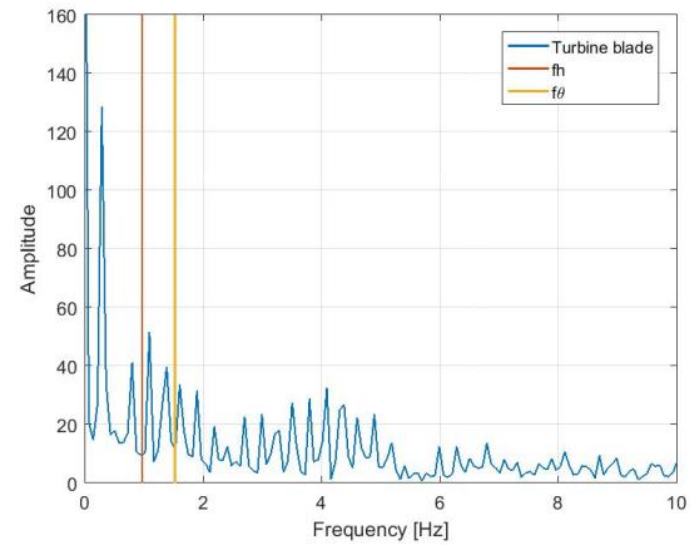

b.

Figure 10. Drag force: a. time dependent, $b$. frequency domain. $f_{h}=$ blade's natural frequency in heave direction, $f_{\theta}=$ blade's natural frequency in pitch direction

Similar to the lift coefficient, the drag coefficient undergoes some fluctuations at one cycle of turbine operation but has different point of location where the maximum and minimum value occur. Lower drag force frequency occurs at the second half of the turbine cycle when the lift force is also relatively low. From Figure 10a, the lower frequency drag force starts at azimuth angle of $326^{\circ}$ and ends at $63^{\circ}$, which is likely caused by wake generation. 


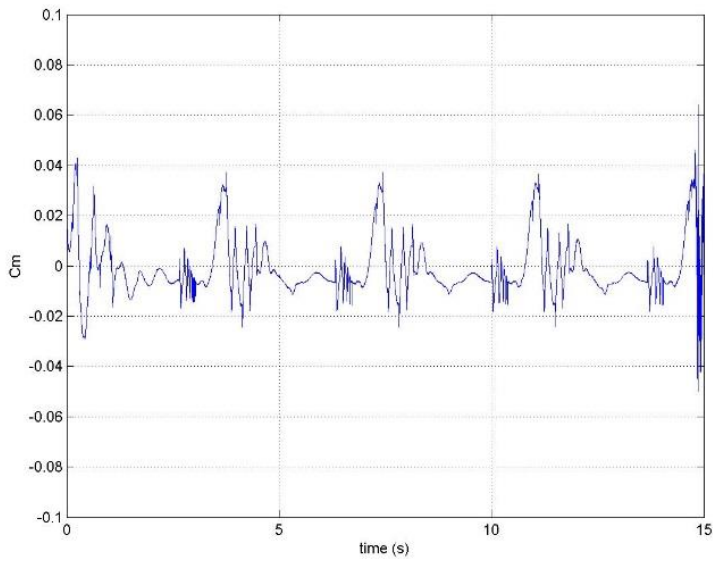

c.

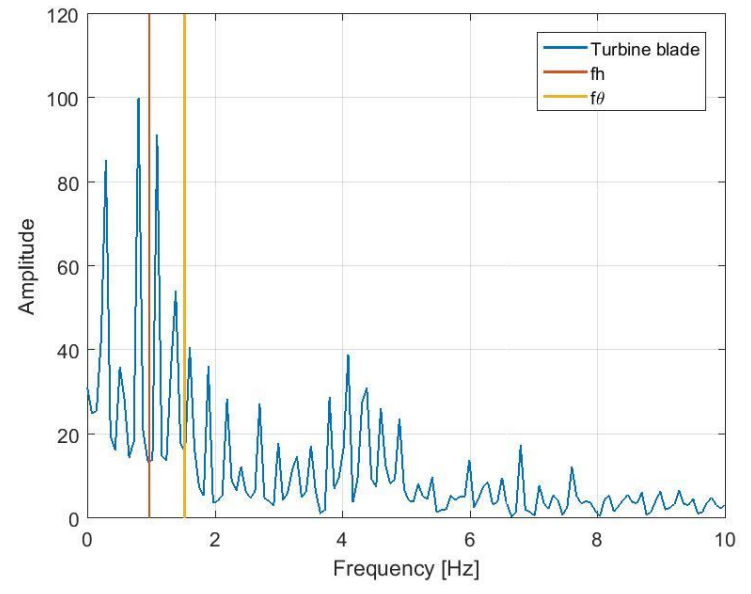

b.

Figure 11. Moment force coefficient: a. time dependent, $b$. frequency domain. $f_{h}=$ blade's natural frequency in heave direction, $f_{\theta}=$ blade's natural frequency in pitch direction

The moment coefficient is recorded over fifteen seconds and is plotted in Figure 11a for time domain plot and 11b for frequency domain plot. As in drag coefficient, the moment coefficient also has some fluctuations during a cycle and the mean value is approximately zero. The maximum moment force happens at about zero azimuth angle when lift force is also maximum which indicates that higher lift force gives more driving force to a turbine blade to rotate. Similar to the drag force, at second half of revolution, the blade experiences higher frequency moment force fluctuations. However there are three different dominant frequencies for moment force as shown in Figure $11 \mathrm{~b}$ which are found to be $0.3,0.8$ and $1.1 \mathrm{~Hz}$.

From Figure 11b, it is also seen that the frequency of $0.8 \mathrm{~Hz}$ and $1.1 \mathrm{~Hz}$ is fairly close to the structural torsional natural frequency of $0.9749 \mathrm{~Hz}$ as explained in section 2.3. It was found that at this condition, a vertical axis tidal turbine is likely to have a resonance and lock-in phenomena which is induced by near wake generation in pitch direction caused by the moment force. This resonance response in pitch direction enhances the blade displacement and creates bigger angle of attack. However this resonance does not occur at all locations over one turbine revolution. It only exists at certain range of azimuth angle which will be detailed in the next section.

\subsection{Near Wake Profile at One Turbine Revolution}

The near wake profile in a vertical axis tidal turbine blade is generated as the effect of angle of attack between resultant fluid velocity and tangential velocity as shown in Figure 3a and blade interaction response. The periodic near wake profile associated with twelve locations on a turbine revolution (azimuth angle) is shown in Figure 12. 


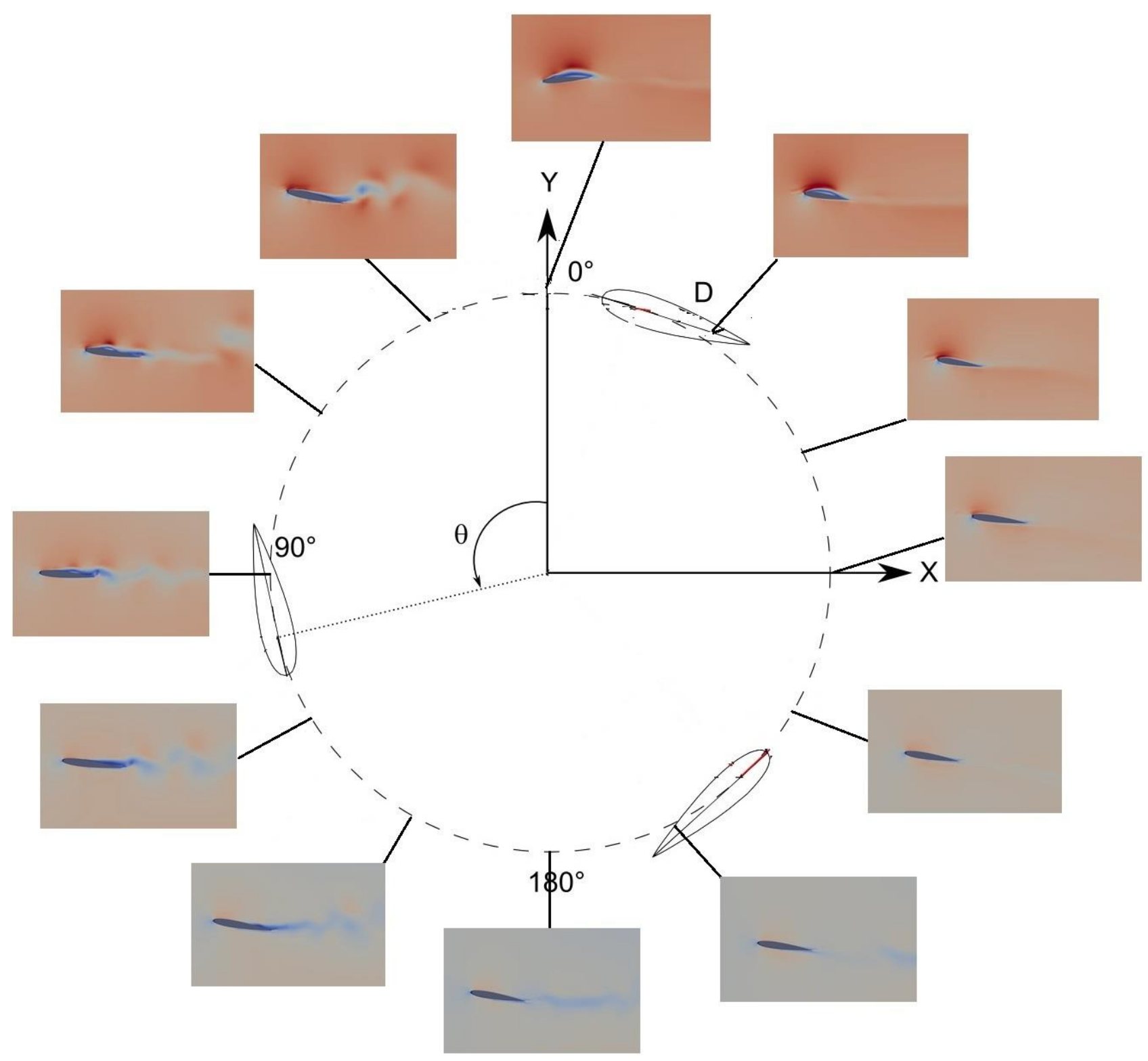

Figure 12. Near wake profile in a vertical axis tidal turbine revolution at azimuth angle of (counterclockwise from $0^{\circ}$ ): $0^{\circ}, 30^{\circ}, 60^{\circ}, 90^{\circ}, 120^{\circ}, 150^{\circ}, 180^{\circ}, 210^{\circ}, 240^{\circ}, 270^{\circ}, 300^{\circ}, 360^{\circ}$.

At the azimuth angle of $0^{\circ}$, wake is generated on the blade outer surface starting from the leading edge and flows towards downstream. From this point the angle of attack increases until it reaches $100^{\circ}$ azimuth angle. Although at that range the incoming velocity decreases, the influence of initial attack is found stronger. Therefore starting at azimuth angle of $30^{\circ}$, the near wake profile is considerably irregular with vortex shedding found behind the trailing edge. This typical fluid regime continues until the blade reaches $120^{\circ}$. The vortex shedding generates fluid-induced vibration on the blade and enhances the response motion as indicated by the high forces coefficient value in Figure 9a, 10a, and 11a. Beyond $120^{\circ}$ azimuth angle, the fluid regime is fairly smooth with small wake formed until the azimuth angle reaches $300^{\circ}$. From this point onward wake generation occurs and the fluid regime development repeats the profile as described above.

\subsection{Pressure Distribution and Blade Displacement}

The fluid structure interaction of a vertical axis tidal turbine in this paper observes the blade vibration which is generated by the tides during a turbine revolution. The vibration is indicated by the blade displacement and can be predicted by the pressure distribution on the blade as depicted in Figure 13. 


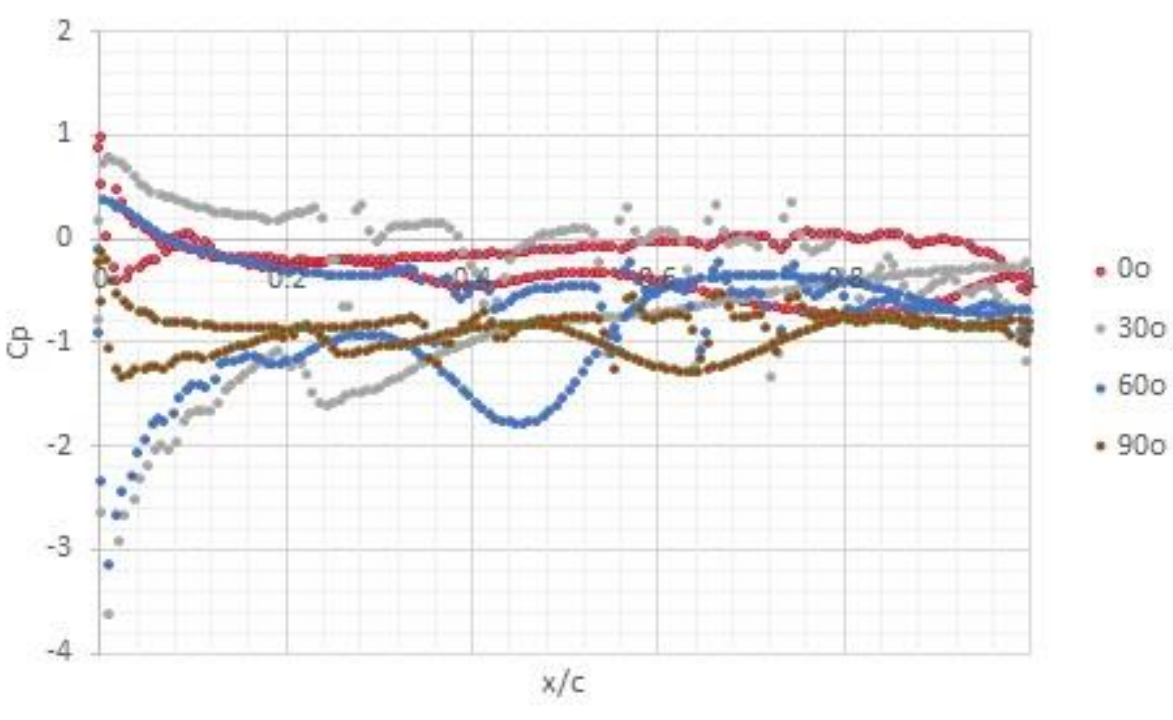

a.

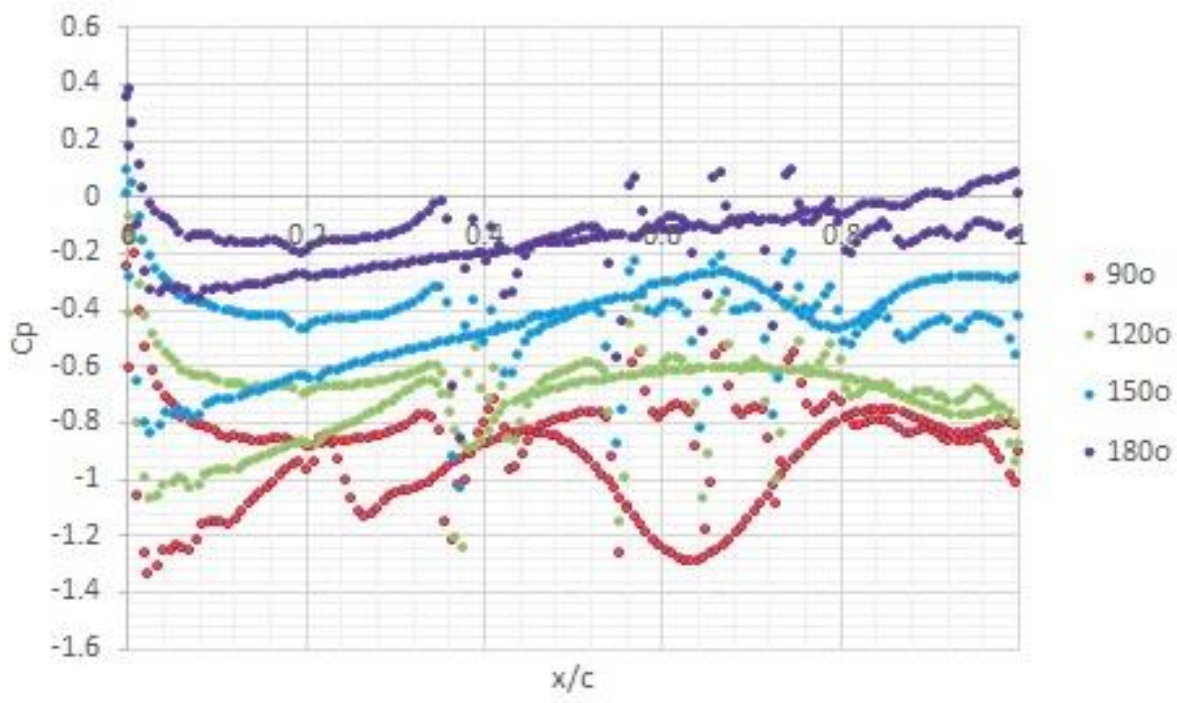

b.

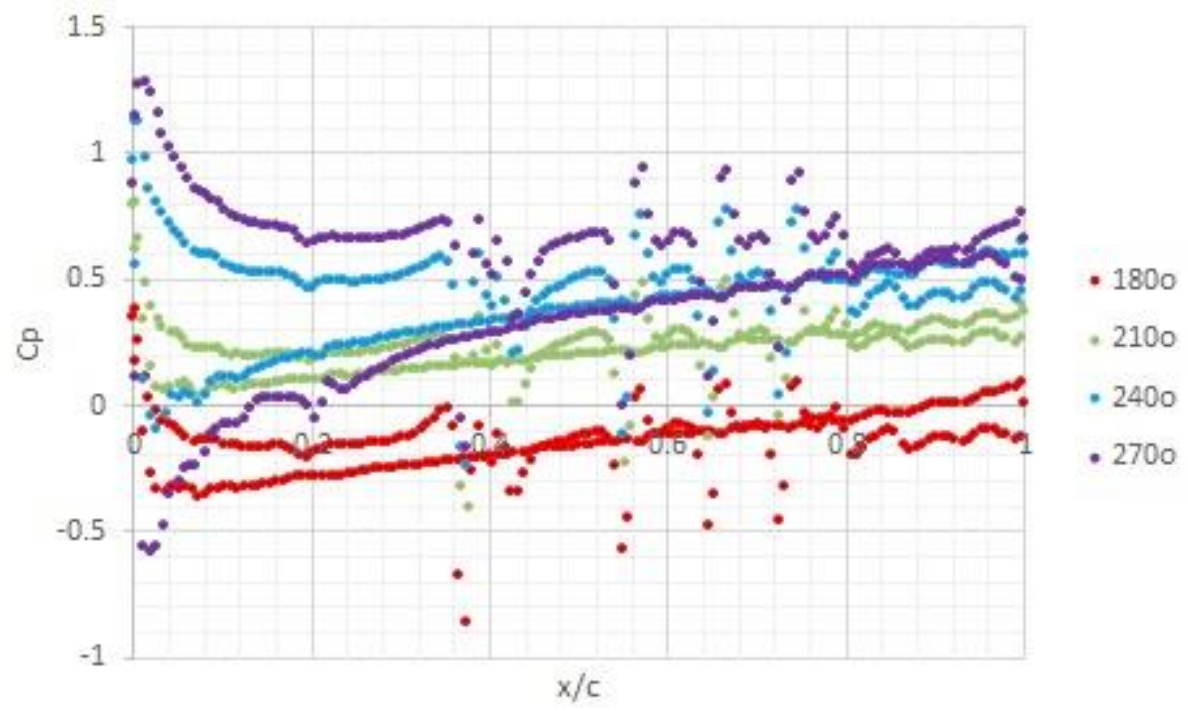

c. 


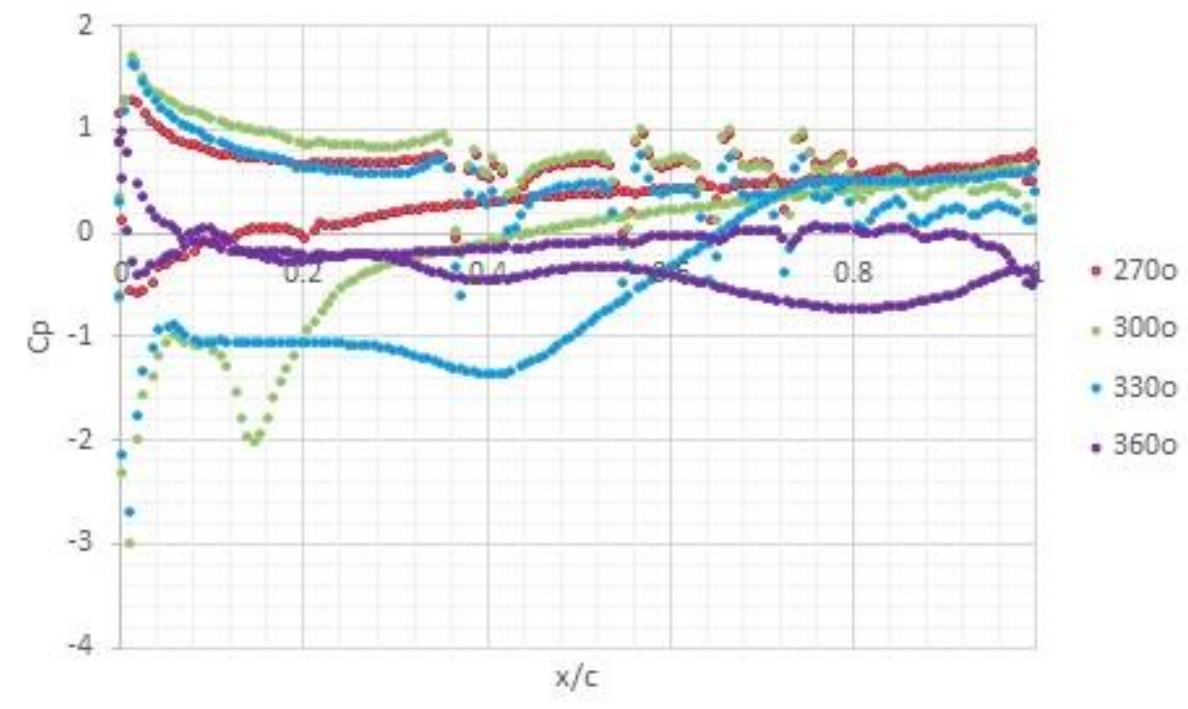

d.

Figure 13. Pressure distribution over one revolution of vertical axis tidal turbine at selected azimuth angle corresponds to Figure 12 : a. $0^{\circ}-90^{\circ}$, b. $90^{\circ}-180^{\circ}$, c. $180^{\circ}-270^{\circ}, 270^{\circ}-360^{\circ}$.

Figure 13 shows pressure distribution plot of a vertical axis tidal turbine fluid flow regime during one revolution starting from $0^{\circ}$ azimuth angle counterclockwise. The plots are shown in every $30^{\circ}$ azimuth angle and depicted in four quadrat sections. The pressure distribution strongly influences the fluid flow regime which is illustrated in Figures 12. At $0^{\circ}$ angle, the vortex is fully detached from the surface as indicated by a flat surface pressure in Figure 13a. A small suction pressure is detected at the leading edge, demonstrated by a vortex creation at that location. At an angle of $30^{\circ}$, formed LEV is moving further along the upper surface which can be seen in the pressure contour plot in Figure 13a. A small peak is found in the plot at $x / c=0.25$ caused by a vortex generation pressure. The LEV grows and flows downward at $60^{\circ}$ azimuth angle marked by higher and receding peak at $x / c=$ 0.46 . The fluid begins to detach and a smaller vortex is attached to the blade surface as shown by a smaller peak at $90^{\circ}$. The peak is located at $x / c=0.65$ which signifies the moving vortices.

The second quadrant pressure distribution which covers the azimuth angle from $90^{\circ}$ to $180^{\circ}$ is shown in Figure $13 \mathrm{~b}$. At $120^{\circ}$ azimuth angle, the vortex is detached from almost the entire upper surface to the free stream and only some attachment is left near the trailing edge. The pressure distribution profile is also shown a constant line at the upper and lower surfaces which indicates that the pressure is almost constant over the entire blade surface. The vortex detachment continues at the azimuth angle of $150^{\circ}$. The separation flow is found on the back of the blade. This is indicated by a small peak at $x / c=0.81$. The vortex is fully detached at $180^{\circ}$ azimuth angle as reflected by a smooth fluid flow regime shown in Figure 12.

The fluid flow regime is consistent with the angle of attack result shown in Figure $8 \mathrm{~b}$ and the velocity result in Figure 8a. In the first quadrant, the velocity decreases, however its magnitude is still high. The high velocity magnitude combined with the increasing angle of attack generates a LEV on the upper surface. The vorticity weakens in the second quadrant due to decreasing velocity and decreasing angle of attack. The fluid flow regime becomes laminar with no vortex detected at $180^{\circ}$ as a result of the low velocity and zero angle of attack.

The pressure distribution contour in the third quadrant of turbine revolution from $180^{\circ}$ to $270^{\circ}$ azimuth angle is shown in Figure 13c. The pressure distribution during this stage are flat with no pressure peak observed. This indicates that no vortex is generated on both surfaces. Although the velocity magnitude increases from the previous condition, the angle of attack acting on the blade decreases. This weakens the effect of the high velocity and prevents the vortex from becoming stronger.

The last quadrant of the revolution is observed at azimuth angles from $270^{\circ}$ to $360^{\circ}$. The pressure distribution associated with the fluid flow regime is shown in Figure $13 \mathrm{~d}$. At $300^{\circ}$ azimuth angle, a suction peak is found at $x / c=0.15$ on the upper surface. This peak indicates vortex initiation at that location as can also be seen in the fluid flow regime image in Figure 12. The vortex flows downstream until the blade reaches $330^{\circ}$ azimuth angle. The moving vortices are also captured in the pressure distribution plot indicated by peak moving away from the leading edge. The vortices generated on this section are weaker than in the first quarter section as shown in the pressure distribution magnitude and the fluid flow regime plot. This is due to the lower angle of attack experienced by the blade. The vortex detaches from the upper surface at $360^{\circ}$ at the first quadrant. The fluid flow condition 
induced by the pressure distribution also generates vibrations on the blade which is indicated by the blade displacement in Figure 14

Figure 14 shows the time history of the blade displacement in $x$ (pointDisplacement 0 ) and $y$ (pointDisplacement 1 ) directions. The displacement of the foil when the blade is at $0^{\circ}$ azimuth angle is taken as a point of vibration reference. At this point the blade is assumed to be in equilibrium position, although this is not the initial non-force condition. The time axis is presented in unit of the time step.

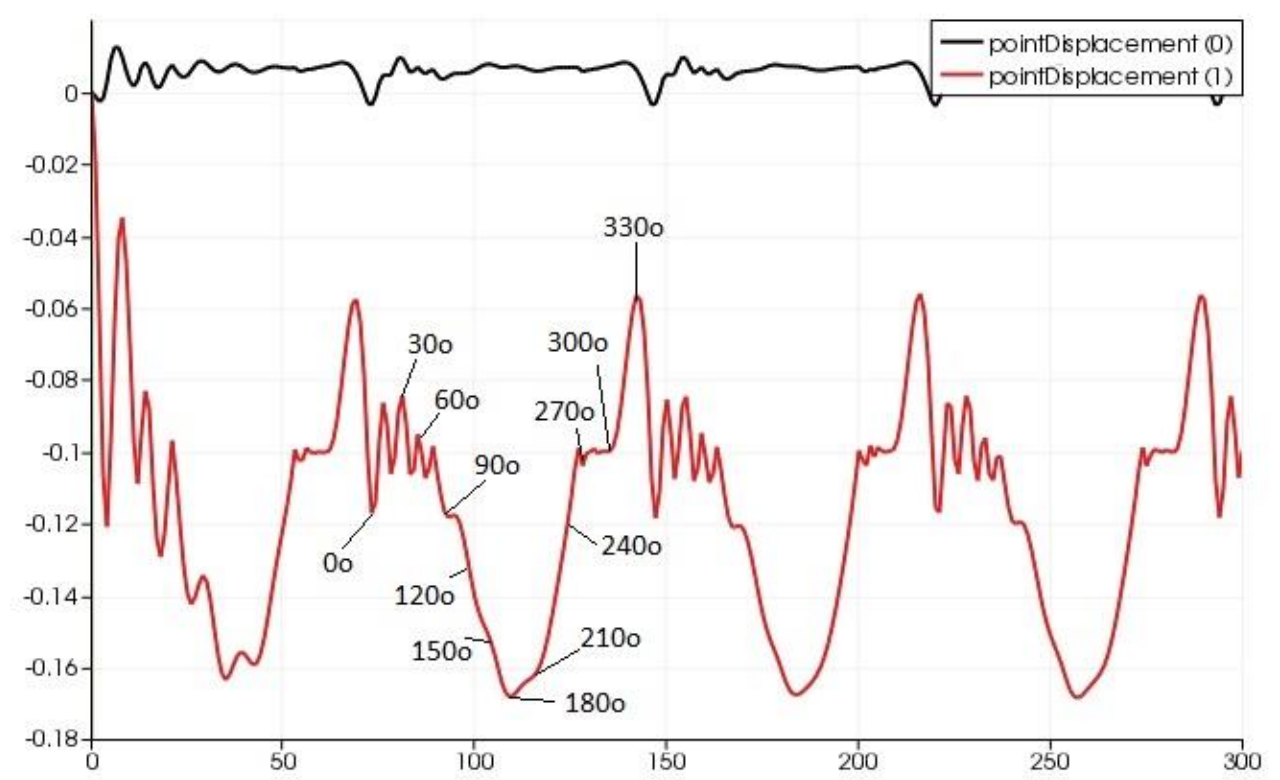

Figure 14. Time history displacement for the original blade response associated with selected azimuth angles, measured at a point of a quarter chord length. pointDisplacement (0): displacement in $\mathrm{x}$ direction, pointDisplacement(1): displacement in y direction

At $0^{\circ}$ azimuth angle, the vortex is fully detached from the surface. The blade surface has no vortex induced vibration acting on it and is at the equilibrium position at this instance. At $30^{\circ}$, an initial LEV begins to form which produces vibrations on the blade, marked by some fluctuations between $0^{\circ}$ and $30^{\circ}$ angle. The upper surface experiences lower pressure which produces more suction pressure on the blade and causes to move to the upper position. From that position, the vortex flows towards the trailing edge and creates further vibrations until it reaches $90^{\circ}$. At this point the pressure on the lower surface decreases and reduces the lift force which causes the blade to move back to its equilibrium position. In the second quarter cycle from azimuth angle of $90^{\circ}$ to $180^{\circ}$, the distribution of the pressure magnitude decreases at all locations over the foil surface. The decreasing pressure reduces the total lift acting on the foil thus the foil moves downwards during this second quarter of the turbine revolution. In the third quarter of revolution, the pressure increases over the foil, thus creating a higher force that moves the blade to its upper position again. In the last quarter of revolution, it can been seen from the distribution pressure contour that total pressure on the blade becomes higher as illustrated by the larger total area inside the upper and lower surface pressure contour. The larger total pressure contributes to a higher force acting on the blade which tends to move the blade to the upper position until it reaches $330^{\circ}$ angle. After that position the pressure becomes lower causing the blade to return to its mid position. The $x$ direction displacement is very low compared to the $y$ direction displacement. This condition is a sign of a weak vibration in $x$ direction acting on the blade. The maximum displacement reaches approximately $0.11 \mathrm{~m}$ and exists at azimuth angle between $68^{\circ}$ and $108^{\circ}$.

\section{Conclusion}

A 2D CFD model of fluid structure interaction on a vertical axis tidal turbine using periodic inflow equivalence model is numerically investigated in this work. The interaction of a vertical axis tidal turbine blade is modelled by a vibration using two spring damper systems and simulated using OpenFOAM 2.2 with sixDoFRigidBodyDisplacement boundary condition to model the vibration response and groovyBC utility to define the periodic inflow equivalence model. Lift, drag and moment forces are observed over twelve seconds and the results are extracted to obtain a frequency domain plot using the FFT method. The frequency domain force coefficients are further used to predict resonant conditions of the turbine structure. The main FSI response frequency of the typical vertical axis tidal turbine blade occurs at 0.8 and $1.1 \mathrm{~Hz}$ which is close to the structural torsional natural frequency of $0.9749 \mathrm{~Hz}$. This situation produces strong vibrations which creates a resonance or lock-in condition.

The lock-in condition is also verified with the pressure distribution and fluid flow regime over one turbine revolution. The pressure distribution on inner and outer surface of the blade are plotted over one turbine rotation. 
The pressure distribution influences vortex generation in the fluid regime. From the result, it is found that the high amplitude vibration response occurs at the second quadrant of the turbine revolution. However the more fluctuated vibration which is likely to generate a resonance or lock-in condition occurs in the first quadrant of revolution which is induced by the strong vortex shedding found in the chaotic fluid regime.

\section{References}

1. Abbot, I. H., Von Doenhoff, A. E. (1959) Theory of wing sections. Dover Publishing, New York

2. Akcabay, D.T et al., 2015a. Cavity induced vibration of flexible hydrofoils. Journal of Fluids and Structures, 49, pp.463-484. Available at: http://dx.doi.org/10.1016/j.jfluidstructs.2014.05.007.

3. Akcabay, D.T. \& Lu, Y., 2015b. Influence of cavitation on the hydroelastic stability of hydrofoils. Journal of Fluids and Structures, 49, pp.170-185. Available at: http://dx.doi.org/10.1016/j.jfluidstructs.2014.04.010.

4. Almohammadi, K. M., Ingham, D. B., Ma, L., Pourkashanian, M. (2015) . 2D CFD Analysis of the effect of trailing edge shape on the performance of a straight blade vertical axis wind turbine.

5. Banks, J., Bercin, K., Lloyd, T. P., Turnock, S. R. (2013) Fluid structure interaction analyses of tidal turbine. $16^{\text {th }}$ numerical towing tank symposium. Mulhemm, Germany.

6. Besem, F. M., Kamrass, J. D., Thomas, J. P., Tang, D., Kielb, R. E. (2015) Vortex Induced Vibration and Frequency Lock-in of an Airfoil at high angle of attack. Journal of Fluids Engineering vol 138

7. Brusca, S., Lanzafame, R., Messina, M (2014). Design of a Vertical Axis Wind Turbine: How the Aspect Ratio Affects The Turbine's Performance. International Journal Energy Environment Engineering 5: 333-340.

8. Chen, X., Zha, G. \& Hu, Z. (2004) Numerical simulation of flow induced vibration based on fully coupled fluidstructural interactions. 34th AIAA Fluid Dynamics Conference.

9. Chung, D. (2001). Review: Material for vibration damping. Journal of materials science, 36(24): 54733-5737.

10. Davies, P., Germain, G., Gaurier, B., Boisseau, A., Perreux, D (2013). Evaluation of the Durability of Composite Tidal Turbine Blades. Proceedings of the royal society of London, February 2013, Volume 371 (1985), pages 115.

11. Ducoin, A. et al. (2009) An experimental and numerical study of the vortices generated by hydrofoils. First International Symposium on Marine Propulsors, (2005).

12. Ducoin, A. \& Young, Y.L. (2013) Hydroelastic response and stability of a hydrofoil in viscous flow. Journal of Fluids and Structures, 38, pp.40-57. Available at: http://dx.doi.org/10.1016/j.jfluidstructs.2012.12.011.

13. Eleni, D. C., Athanasios, T. I., Dionissios, M. P. (2012) Evaluation of the turbulence models for the simulation of the flow over a NACA 0012 airfoil.Journal of Mechanical Engineering Research Vol 4(3) pp. 100-111.

14. Gosselin, R., Dumas, G., Boudreau, M. (2013) Parametric study of H-Darrieus vertical axis tidal turbines using uRANS simulations. $21^{\text {st }}$ annual conference of the CFD society of Canada.

15. Gretton, G. I Gretton, G. I., Bruce, T., Ingram, D. M. (2009) Hydrodynamic modelling of a vertical axis tidal current turbine using CFD. $8^{\text {th }}$ European wave and tidal conference (EWETEC 2009).

16. Khalid, S., Zhang, L., Zhang, X., Sun K. (2013) Three dimensional numerical simulation of a vertical axis tidal turbine using the two way fluid structure interaction approach. Journal of Zheijang University-SCIENCE (apllied physics of engineering) $14(8): 574-582$

17. Kumar, D. P. S. (2015). Karthik. k and Raja. T. vibration damping characteristics of hybrid polymer matrix composite. International Journal of Mechanical \& Mechatronics Engineering IJMME-IJENS, 15(01):153101-8282.

18. Lanzafame, R., Mauro, S., Messina, M. (2014) 2D CFD modelling of H-Darrieus wind turbine using a transition turbulence model. $68^{\text {th }}$ conference of Italian thermal machines engineering association, ATAI2013. Energy Procedia 45, pp 131-140.

19. Lee, T. \& Su, Y.Y., (2015). Surface Pressures Developed on an Airfoil Undergoing Heaving and Pitching Motion. Journal of Fluids Engineering, 137(5), pp.1-11. Available at: http://fluidsengineering.asmedigitalcollection.asme.org/article.aspx?doi=10.1115/1.4029443

20. Li, Y. \& Calisal, S.M., (2010). Numerical analysis of the characteristics of vertical axis tidal current turbines. Renewable Energy, 35(2), pp.435-442. Available at: <Go to ISI>://WOS:000271688300015.

21. Lundin, M. G. U., Leijon, M. (2010) Ocean Energy. Research Report. Department of Electricity and Lightning Research Uppsala University, Sweden.

22. OpenFoam foundation (2016) the open source CFD Toolbox, user guide, Open $\nabla$ Foam. Availabel from: http://www.openFoam.org/docs/user/tutorials.php [accessed March 31, 2014]

23. Rana, D. et al. (2011) Time Domain Simulation of Airfoil Flutter Using Fluid Structure Coupling Through Fem Based Cfd Solver. Symposium on Applied Aerodynamic and Design of Aerospace Vehicle, (Sarod).

24. Rouke, O. F., Boyle, F., Reynolds, A. (2010) Tidal Energy Update 2009. Applied Energy 87 (2010), 398-409. 
25. Tabassian, R. (2013). Torsional vibration analysis of shafts based on adomian decomposition method. Applied and Computational Mechanics 7, 205-222.

26. Tillinger, D., 2011. Physical oceanography of the present day Indonesian Throughflow. Geological Society, London, Special Publications, 355(1), pp.267-281. Available at: http://sp.lyellcollection.org/content/355/1/267.abstract.

27. Veilleux, J. C., Dumas, G. (2013). Numerical simulations of experimentally observed high-amplitude selfsustained pitch-heave oscillations of a NACA 0012 airfoil. 21 $1^{\text {st }}$ Annual conference of the CFD Society of Canada.

28. Young, Y.L., Motley, M.R. \& Yeung, R.W. (2010). Three-Dimensional Numerical Modeling of the Transient FluidStructural Interaction Response of Tidal Turbines. Journal of Offshore Mechanics and Arctic Engineering, 132(1), p.11101. Available at: http://offshoremechanics.asmedigitalcollection.asme.org/article.aspx?articleid=1472752. 